MATEC Web of Conferences 44, 02012 (2016)

DOI: $10.1051 /$ matecconf/20164402012

(C) Owned by the authors, published by EDP Sciences, 2016

\title{
Guided Waves in a Solid Rod Embedded in Infinite Medium with Weak Interface
}

\author{
Zi Wei Tong ${ }^{1}$, Qing Bang Han ${ }^{1,2,{ }^{*}}$, Cheng Yin ${ }^{1}$, Li Hua Qi ${ }^{1}$, Yu Zhang ${ }^{1}$ and Chang Ping Zhu ${ }^{1}$ \\ 1 College of IOT Engineering, Hohai University, Changzhou 213022, China \\ 2 Huaian Research Institute, Hohai University, Huaian 223001, China
}

\begin{abstract}
The dispersion relation of the infinite long rod model with a weak interface has been deduced via wave equation of the guided waves and boundary spring interface model. The dispersion characteristics of the rigid interface and the slip interface are calculated, while the effect of different axial stiffness coefficient is also discussed. The results suggest that the phase velocity of the slip interface decreases slowly with increasing frequency at the low frequency of the L $(0,1)$ mode. As for the weak interface, when the axial coefficient $\mathrm{Kt}$ is in the particular range of $\left[10^{10}, 10^{14}\right] \mathrm{pa} / \mathrm{m}$, the interface turns from the slip interface to the rigid interface. That shows the value of axial coefficient $\mathrm{Kt}$ is an essential prerequisite for the non-destructive evaluation to evaluate the interface cementation degree.
\end{abstract}

\section{Introduction}

Propagation of guided waves in a solid rod embedded in infinite medium with weak interface is an interesting research topic of practical importance. For example, it is urgent to apply Non-destructive evaluation (NDE) for the rock bolts in the mixture of rock and concrete, which are inserted to ensure the stability of the groundwork. One key issue in the above mentioned NDE process is how to evaluate bonding state between rock bolt and concrete by guided ultrasonic waves ${ }^{[1-4]}$.

The propagation of guided wave in solid cylindrical media has already been widely studied, but most work is based on a model of a multilayered columnar structure with continuous interface ${ }^{[5-8]}$, few attention has been focused on the semi-infinite space structure with discontinuous interface ${ }^{[9-10]}$.
In this paper, we study the propagation characteristics of guided waves in a solid rod embedded in infinite medium with weak interface. This paper is arranged as follows. First, the dispersion equation using spring interface model is deduced. Second, the dispersion characteristics of guided waves in the structure under the rigid interface and slip interface are discussed, respectively. Third, we investigated the effects of different axial coefficient on the guided waves under the weak interface.

\section{Theory}

\subsection{Weak Interface}

The interface between different solid media of the layered structure is inevitable, and can be divided into two

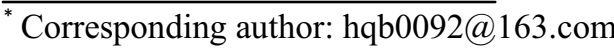


categories, i.e., the rigid interface and the slip interface. The rigid interface can be presented as an interface that usually satisfies the boundary conditions of continuous stress and displacement of elastic waves while two solid medium components are well cemented. And the slip interface ${ }^{[7]}$ is such an interface that can withstand the normal (or radial) stress perpendicular to the interface while the tangential (or axial) stress is identical to zero. That is, the normal (or radial) stress and displacement are continuous; the tangential (or axial) displacement is discontinuity $^{[11]}$.

In the procedure of using complex media or composite material, the micro-cracks occur at the interface, and the mechanical strength is reduced to form a weak boundary layer. The spring model can describe the physical characteristics of a weak interface, thus the boundary conditions of mechanical quantity at the interface is available ${ }^{[11]}$.

\subsection{Dispersion Equation}

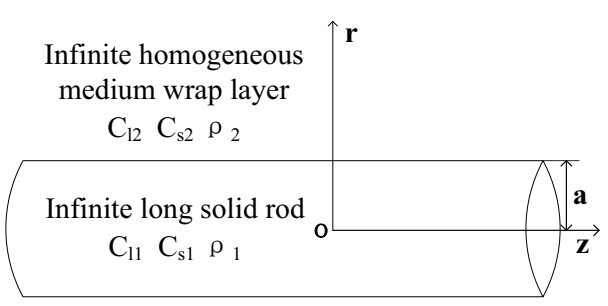

Figure 1. The schematic of infinite long solid rod embedded in infinite homogeneous medium

The system of this paper is modelled as an infinite long solid rod embedded in an infinite homogeneous isotropic medium as shown in Fig.1. It is convenient to solve this problem using cylindrical coordinate, where the $\mathrm{z}$ axis of the coordinate is the central axis of the solid rod. In this paper, only longitudinal modes are taken into consideration. $\varphi 1, \psi 1, \chi 1$ and $\varphi 2, \psi 2, \chi 2$ are used to represent the potential function of solid rod and wrap layer. The potential function can be expressed as

$$
\begin{gathered}
\left\{\begin{array}{c}
\varphi_{1}=A_{1} I_{n}\left(\alpha_{1} \mathrm{r}\right)+K_{n}\left(\alpha_{1} r\right) \\
\psi_{1}=C_{1} I_{n}\left(\beta_{1} \mathrm{r}\right)
\end{array}\right. \\
\left\{\begin{array}{l}
\varphi_{2}=B_{2} K_{n}\left(\alpha_{2} r\right) \\
\psi_{2}=D_{2} K_{n}\left(\beta_{2} r\right)
\end{array}\right.
\end{gathered}
$$

The potential function satisfies the wave equation, and can be described as

$$
\begin{aligned}
& \nabla^{2} \Phi=\frac{1}{\mathrm{C}_{l}^{2}} \frac{\partial^{2} \Phi}{\partial \mathrm{t}^{2}} \\
& \nabla^{2} \Psi=\frac{1}{\mathrm{C}_{\mathrm{s}}^{2}} \frac{\partial^{2} \Psi}{\partial \mathrm{t}^{2}}
\end{aligned}
$$

where, $\mathrm{Cl}$ and $\mathrm{Cs}$ are the $\mathrm{P}$-wave velocity and $\mathrm{S}$-wave velocity, respectively. $\mathrm{t}$ is time, and $\nabla^{2}$ is Laplace operator.

$$
\begin{gathered}
\mathrm{C}_{l}^{2}=\frac{\lambda+2 \mu}{\rho} \\
\mathrm{C}_{\mathrm{s}}^{2}=\frac{\mu}{\rho} \\
\nabla^{2}=\frac{\partial^{2}}{\partial \mathrm{r}^{2}}+\frac{1}{\mathrm{r}} \frac{\partial}{\partial \mathrm{r}}+\frac{\partial^{2}}{\partial \mathrm{z}^{2}}
\end{gathered}
$$

Here, $\lambda$ and $\mu$ are both Lame parameters, $\rho$ is the density.

It is convenient to introduce displacement potentials.

$$
\left\{\begin{array}{c}
\mathrm{u}_{\mathrm{r}}=\frac{\partial \Phi}{\partial \mathrm{r}}+\frac{\partial^{2} \Psi}{\partial \mathrm{r} \partial \mathrm{z}} \\
\mathrm{u}_{\mathrm{z}}=\frac{\partial \Phi}{\partial \mathrm{z}}-\frac{\partial^{2} \Psi}{\partial \mathrm{r}^{2}}-\frac{1}{\mathrm{r}} \frac{\partial \Psi}{\partial \mathrm{r}}
\end{array}\right.
$$

The normal and tangential stress tractions are related to displacements using the Hooke's law.

$$
\left\{\begin{array}{c}
\sigma_{\mathrm{rr}}=2 \mu \frac{\partial \mathrm{u}_{\mathrm{r}}}{\partial \mathrm{r}}+\lambda\left(\frac{\mathrm{u}_{\mathrm{r}}}{\mathrm{r}}+\frac{\partial \mathrm{u}_{\mathrm{r}}}{\partial \mathrm{r}}+\frac{\partial \mathrm{u}_{\mathrm{z}}}{\partial \mathrm{z}}\right) \\
\sigma_{\mathrm{rz}}=\mu\left(\frac{\partial \mathrm{u}_{\mathrm{r}}}{\partial \mathrm{z}}+\frac{\partial \mathrm{u}_{\mathrm{z}}}{\partial \mathrm{r}}\right)
\end{array}\right.
$$

The radial and axial displacement components ur and uz can be expressed by substituting Eqs.(1) and (2) into Eq.(8).The stress components $\sigma_{\mathrm{rr}}$ and $\sigma_{\mathrm{rz}}$ in Eq. (9) also can be obtained.

A spring interface model is established in cylindrical interface $(r=a)$. Based on the model, the boundary conditions can be written as

$$
\left\{\begin{array}{l}
\sigma_{r r}^{(1)}=\operatorname{Kn}\left(u_{r}^{(1)}-u_{r}^{(2)}\right) \\
\sigma_{r z}^{(1)}=\operatorname{Kt}\left(u_{z}^{(1)}-u_{z}^{(2)}\right) \\
\sigma_{r r}^{(1)}=\sigma_{r r}^{(2)} \\
\sigma_{r z}^{(1)}=\sigma_{r z}^{(2)}
\end{array}\right.
$$

where, $\mathrm{Kn}$ and $\mathrm{Kt}$ are the radial stiffness coefficient, and the axial stiffness coefficient, respectively. The functions of $\mathrm{Kn}$ and $\mathrm{Kt}$ are composed of the interface layer thickness $\mathrm{h}$, equivalent bulk modulus $\mathrm{K} 0$ and shear modulus $\mathrm{G} 0^{[11]}$. They are expressed as

$$
\begin{gathered}
\mathrm{Kn}=\left(\mathrm{K}_{0}+\frac{4}{3} \mathrm{G}_{0}\right) / h \\
\mathrm{Kt}=\mathrm{G}_{0} / h
\end{gathered}
$$


The boundary condition of rigid interface and slip interface can be deduced from the Eq.(10). When $K n, K t \rightarrow \infty$, the stress is finite, so $\mathrm{u}_{r}^{(1)}=u_{r}^{(2)}, \mathrm{u}_{z}^{(1)}=u_{z}^{(2)}$, equivalent to the continuous boundary condition of rigid interface. When $K n \rightarrow \infty, K t=0$, the boundary condition is $\mathrm{u}_{r}^{(1)}=u_{r}^{(2)}, \sigma_{r z}^{(1)}=\sigma_{r z}^{(2)}=0$, which is equivalent to the boundary condition of slip interface. It can be seen that the axial elasticity coefficient is used as a measuring metric of the axial stress tolerance level as well as an essential parameter describing a weak interface.

By substituting displacement and stress components into Eq.(10), the equations can be written as,

$$
\left[\begin{array}{llll}
m_{11} & m_{12} & m_{13} & m_{14} \\
m_{21} & m_{22} & m_{23} & m_{24} \\
m_{31} & m_{32} & m_{33} & m_{34} \\
m_{41} & m_{42} & m_{43} & m_{44}
\end{array}\right]\left[\begin{array}{l}
A_{1} \\
C_{1} \\
B_{2} \\
D_{2}
\end{array}\right]=\left[\begin{array}{l}
b_{1} \\
b_{2} \\
b_{3} \\
b_{4}
\end{array}\right]
$$

When the right-hand side of Eq.(13) is zero matrix, the necessary and sufficient condition for the non-zero solutions of the equation is the coefficient determinant $\Delta=0$. It is the dispersion equation whose solution corresponds to the guided wave.

For given different values of $\mathrm{Kn}$ and $\mathrm{Kt}$, solutions for this dispersion equation can be got which yield the correspondence between the phase velocity and frequency under the rigid interface, slip interface and weak interface.

\section{Numerical results}

Since our work aims at the rock bolt detection, our simulation selects concrete as material of infinite medium, and steel as the solid rod. By setting the radius of solid rod to be $10 \mathrm{~mm}$ so that the dispersion characteristics of the structure under rigid interface, slip interface and different axial stiffness coefficient are analysed.

\subsection{Rigid Interface}

As the stiffness coefficient $K n, K t \rightarrow \infty$, the interface is the rigid interface, i.e., the stress and displacement are continuous.

The phase velocity dispersion curves are shown in the Fig.2. As can be seen, the phase velocity of the lowest mode gradually approaches to the shear-wave velocity $(3260 \mathrm{~m} / \mathrm{s})$ of the steel with increasing frequency. In each mode, the phase velocity decreases as the frequency gradually increases. Another feature is that each higher-order mode has a cut off frequency.

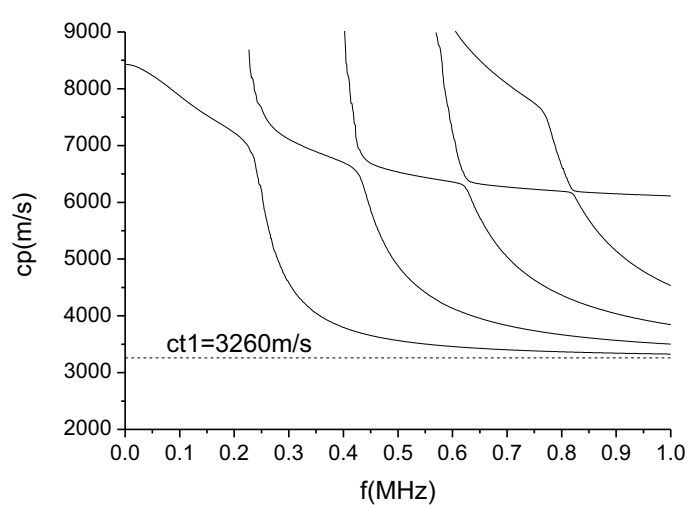

Figure2. Phase velocity dispersion curve under rigid interface

\subsection{Slip Interface}

When the stiffness coefficient $K n \rightarrow \infty, K \mathrm{t}=0$, the radial displacement and axial stress are continuous. The boundary conditions are $\mathrm{u}_{r}^{(1)}=u_{r}^{(2)}, \sigma_{r z}^{(1)}=\sigma_{r z}^{(2)}=0$, which is slip interface.

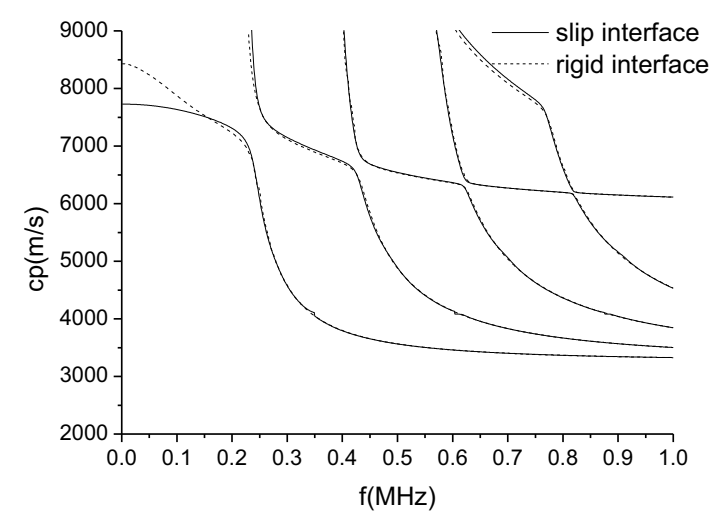

Figure 3. Dispersion curves under slip interface and rigid interface

In Fig.3, we show the phase velocity dispersion curves obtained under these boundary conditions. Several features of these results that different from rigid interface and slip interface deserve comment, especially in the low frequency of the first mode. When the frequency of the $\mathrm{L}(0,1)$ mode is in $[0,0.2] \mathrm{MHz}$, the phase velocity of the slip interface decreases more slowly than that in the rigid interface as the frequency increases. However, in the high-order modes, there is no significant difference between the two dispersion curves.

\subsection{Weak Interface}


As for the spring model, the axial elasticity coefficient is a measure of weak interface to withstand axial stress. Therefore, the dispersion curves under different axial stiffness coefficient are calculated to evaluate the weak interface.

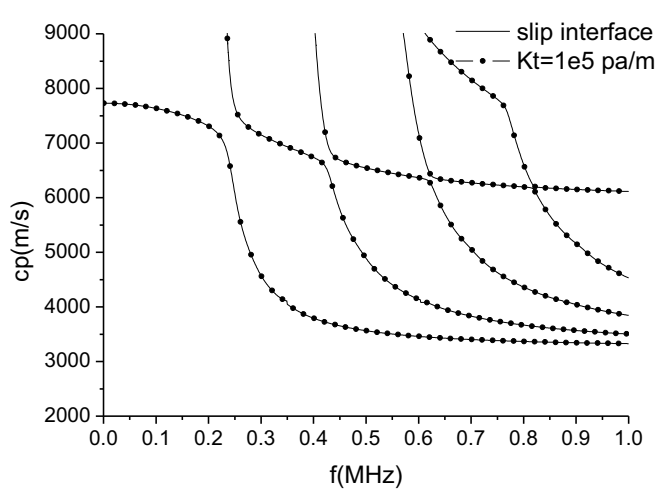

Figure 4. Dispersion curves under slip interface and $K n \rightarrow \infty, \mathrm{Kt}=10^{5} \mathrm{pa} / \mathrm{m}$

Fig.4 shows the dispersion curves achieved by deducing the dispersion equation under the boundary condition $K n \rightarrow \infty, K \mathrm{t}=10^{5} \mathrm{pa} / \mathrm{m}$. It can be found that the phase velocity dispersion curves are consistent with the dispersion curves under slip interface.

In Figure 5, we show the dispersion curves under the boundary conditions $K n \rightarrow \infty, K \mathrm{t}=10^{19} \mathrm{pa} / \mathrm{m}$. Phase velocity dispersion curves under this case are similar with the dispersion curves under rigid interface.

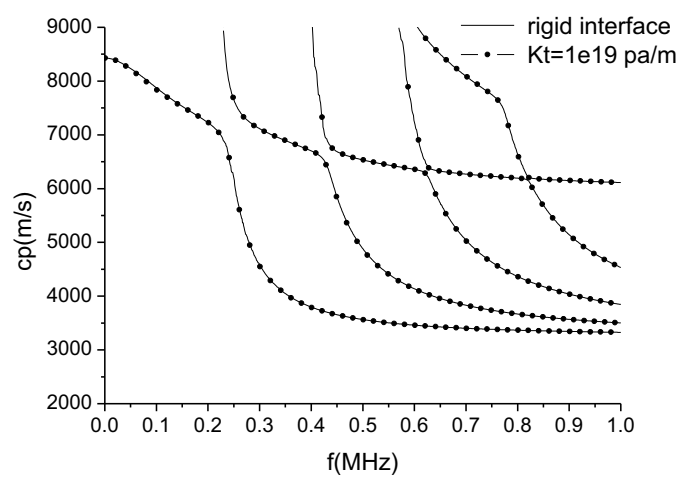

Figure 5. Dispersion curves under rigid interface a $\mathrm{Kn} \rightarrow \infty, \mathrm{Kt}=10^{19} \mathrm{pa} / \mathrm{m}$

Since only two cases concerned, it's not accurate to explain the impact brought by the axial stiffness coefficient on the guided waves. By setting $\mathrm{f}=0.1 \mathrm{MHz}$, $K n \rightarrow \infty$, the phase velocity of $\mathrm{L}(0,1)$ mode respect to the axial stiffness coefficient are shown in Fig.6. When $\mathrm{Kt}$ is in $\left[10^{5}, 10^{10}\right] \mathrm{pa} / \mathrm{m}$ and $\left[10^{14}, 10^{19}\right] \mathrm{pa} / \mathrm{m}$, the phase velocity remains unchanged. While $\mathrm{Kt}$ is in the particular value range of $\left[10^{10}, 10^{14}\right] \mathrm{pa} / \mathrm{m}$, the interface turns from the slip interface to the rigid interface, and the phase velocity is sensitive to the changes of $\mathrm{Kt}$ as well. When $\mathrm{Kt}$ is higher than $10^{10} \mathrm{pa} / \mathrm{m}$, the phase velocity starts decreasing gradually as $\mathrm{Kt}$ increases, and stays at a minimum value when $\mathrm{Kt}$ approaches $10^{12} \mathrm{pa} / \mathrm{m}$.

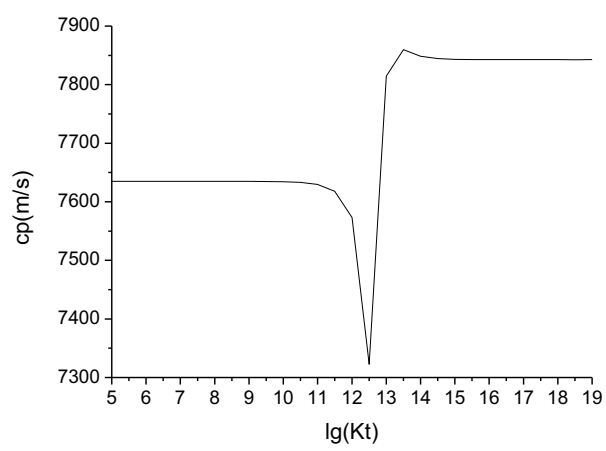

Figure 6. Guided wave phase velocity curve with $\mathrm{Kt}$ when $\mathrm{f}=0.1 \mathrm{MHz}$

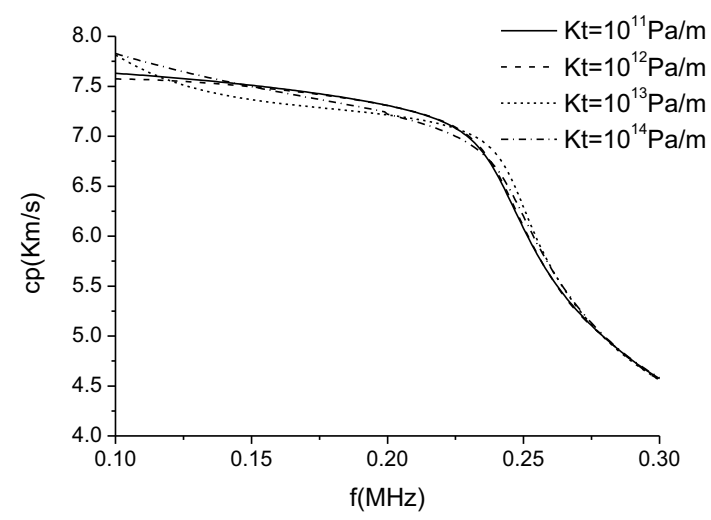

Figure 7. Guided wave phase velocity curve of $\mathrm{L}(0,1)$ mode with different $\mathrm{Kt}$

The dispersion curves of $\mathrm{L}(0,1)$ mode with $\mathrm{Kt}=$ $10^{11} \mathrm{pa} / \mathrm{m}, 10^{12} \mathrm{pa} / \mathrm{m}, 10^{13} \mathrm{pa} / \mathrm{m}, 10^{14} \mathrm{pa} / \mathrm{m}$ are shown in Fig.7. The main reason for choosing is that the phase velocity of $\mathrm{L}(0,1)$ mode varies greatly when the axial coefficient $\mathrm{Kt}$ is in the range of $\left[10^{10}, 10^{14}\right] \mathrm{pa} / \mathrm{m}$. Fig.7 shows that the dispersion curves for $\mathrm{Kt}=10^{13} \mathrm{pa} / \mathrm{m}$ and $\mathrm{Kt}=10^{14} \mathrm{pa} / \mathrm{m}$ have more obvious fluctuations than the dispersion curves for $\mathrm{Kt}=10^{11} \mathrm{pa} / \mathrm{m}$ and $\mathrm{Kt}=10^{12} \mathrm{pa} / \mathrm{m}$ when the frequency is smaller than $0.3 \mathrm{MHz}$. As the axial coefficient increases, the phase velocity decreases faster with the frequency increasing.

From the above analysis, the dispersion curves on the $\mathrm{L}(0,1)$ mode of this structure have much more significant changes in a certain range of Kt. Therefore, in non-destructive evaluation, the interface cementation degree can be fully evaluated through measuring the 
phase velocity of the guided wave propagating in the structure and calculating the value of $\mathrm{Kt}$ by inversion.

\section{Conclusions}

Based on the analysis, the phase velocity of the lowest mode converges to the shear-wave velocity of the solid rod with increasing frequency. Numerical results validate that, compared to rigid interface, the phase velocity of the slip interface decreases slowly with increasing frequency at the low frequency of the $\mathrm{L}(0,1)$ mode. As for the weak interface, when the axial coefficient $\mathrm{Kt}$ is in the particular range of $\left[10^{10}, 10^{14}\right] \mathrm{pa} / \mathrm{m}$, the interface transfers from the slip interface to the rigid interface and the phase velocity shows sensitivity to any slight change of the axial coefficient $\mathrm{Kt}$. The value of axial coefficient $\mathrm{Kt}$ is an essential prerequisite for the non-destructive evaluation to evaluate the interface cementation degree.

There are still a lot of works to do in the future. One scope is the simulation of guided wave propagation in such structure model. We can establish the model with different level of split at the interface, research the propagation characteristic of the guided wave, and calculate the value of $\mathrm{Kt}$ by inversion according to the numerical results of simulation. Finally, the result of simulation would be useful to the non-destructive evaluation.

\section{Acknowledgements}

This work is supported by the Natural Science foundation of China Grant No. 11274091, 11574072 and the Fundamental Research Funds for the Central Universities of Hohai University No:2011B11014.

\section{References}

[1] Ivanovic A, Neilson R D, "Influence of geometry and material properties on the axial vibration of a rock bolt," Int. J. Rock Mech. Mining Sci.,45:941-951(2008).

[2] Madenga V, Zou D H, Zhang C., "Effects of curing time and frequency on ultrasonic wave velocity in grouted rock bolts ,J. Appl.Geophys.,59:79-87(2006).
[3] M. D. Beard and M. J. S. Lowe, "Non-destructive testing of rock bolts using guided ultrasonic waves," Int. J. Rock Mech. Min. Sci. 40, 527-536(2003).

[4] M. D. Beard, M. J. S. Lowe, and P. Cawley, "Inspection of rock bolts using guided ultrasonic waves," in Review of Progress in QNDE, edited by D.O.Thompson and D. E. Chimenti, 20, pp.1156-1163(2001).

[5] B. X. Zhang and H. Y. Cui, "Guided waves in a multi-layered cylindrical elastic solid medium," 1 . Chin. Phys. Lett.,24(10): pp. 2883-2886(2007).

[6] H. Y. Cui and B. X. Zhang, Sherri Johnstone and Jon Trevelyan, "Excitation mechanisms and dispersion characteristics of guided waves in multilayered cylindrical solid media," J. Acoust. Soc. Am.,131 (3):pp.2048-2062(2012)

[7] L. Laguerre and A. Grimault, "Ultrasonic transient bounded-beam propagation a solid cylinder waveguide embedded in a solid medium,"J.Acoust.Soc.Am.,121(4),1924-1934 (2007).

[8] A.D. Puckett and M. L. Peterson, "A semi-analytical model for predicting multiple propagating axially symmetric modes in cylindrical waveguides,'Ultrasonics,43, 197-207(2005).

[9] Elena Maeva, Inna Severina, Sergiy Bondarenko, et al. "Acoustical methods for the investigation of adhesively bonded structures," A Review. Can J Phys., 82:981-1025(2004).

[10] A.Baltazar, L.Wang, B.Xie, et al. "Inverse ultrasonic determination of imperfect interfaces and bulk properties of a layer between two solids," J.Acoust.Soc.Am,114(3):1424-1434(2003).

[11] Zhang Rui, Wang Mingxi, Cao Wenwu,"Guided Wave in ultra-thin layered composite structure with weak and slip interface in different depth," Chin .Phys .Soc., 49(7):1297-1302(2000). 